INGENIERÍA DE MATERIALES

\title{
Crecimiento de nanotubos de carbono sobre el mineral limonita como catalizador
}

MATERIALS ENGINEERING

\section{Growth of carbon nanotubes over Colombian natural limonite ore as catalysts}

\author{
$\S$ Diana M Torres *, Germán A Sierra* \\ * Escuela de Ingeniería de Materiales, Universidad Nacional de Colombia. Medellín, Colombia \\ §dmtorresho@unal.edu.co,geasierraga@unal.edu.co
}

(Recibido: Diciembre 14 de 2011 - Aceptado: Noviembre 19 de 2012)

\begin{abstract}
Resumen.
Nanotubos de carbono multicapa (MWCNTs) fueron sintetizados usando metano como fuente de carbono y el mineral colombiano limonita como precursor del catalizador. El catalizador fue evaluado en forma reducida en la descomposición del metano a $700^{\circ} \mathrm{C}$. Los nanotubos de carbono obtenidos fueron del tipo multicapa, los cuales fueron caracterizados con Difracción de rayos X (DRX), Microscopía electrónica de transmisión (TEM), microscopía electrónica de barrido (SEM) y análisis termogravimétrico (TGA). Las micrografías TEM mostraron que los MWCNTs poseen diámetros internos que van desde 5 hasta $30 \mathrm{~nm}$ y diámetros externos de hasta $60 \mathrm{~nm}$ y varias micras de longitud.
\end{abstract}

Palabras clave: Descomposición de metano, Limonita, Nanomateriales, Nanotubos de carbono.

\begin{abstract}
.
Multi-walled carbon nanotubes were synthesized using methane as carbon source and Colombian natural limonite ore as catalyst precursor. Catalyst was evaluated in reduced form in the reaction decomposition of methane at $700^{\circ} \mathrm{C}$.

Multi walled carbon nanotubes were characterized by X-ray diffraction (XRD), transmission electron microscopy (TEM), scanning electron microscopy (SEM) and thermogravimetric analysis (TGA). Finally, TEM micrographs showed that the MWCNTs have inner diameters ranging from 5 to $30 \mathrm{~nm}$ and outer diameters up to about $60 \mathrm{~nm}$, and several microns in length.
\end{abstract}

Keywords: Carbon nanotubes, Colombia's Limonite ores, Nanomaterials, Methane decomposition. 


\section{Introducción}

Los minerales basados en hierro ocupan el $4^{\circ}$ lugar en abundancia entre todos los elementos en la corteza terrestre. Entre los más abundantes están la magnetita, siderita y limonita. La limonita es un mineral natural compuesto por óxidos hidratados de hierro (III) en su mayoría goethita, su contenido de hierro está entre el 20 y el $45 \%$ y su fórmula genérica es frecuentemente escrita como $\mathrm{FeO}(\mathrm{OH}) \cdot \mathrm{nH}_{2} \mathrm{O}$.

Este mineral es una atractiva alternativa como catalizador por su alto contenido en hierro porque no es costoso y es un recurso abundante Souza et al. (2007).

Se han realizado muchos estudios utilizando como catalizador la limonita, por ejemplo en la licuefacción directa de carbón Kaneko et al. (2002), la descomposición de amoniaco Tsubouchi et al. (2008), la oxidación orgánica, hidrocraqueo Matsumura et al. (2005), entre otros; sin embargo, no hay ningún reporte de su potencial catalítico para el crecimiento de nanotubos de carbono.

El término nanotubo de carbono se relaciona normalmente con una estructura con los planos basales del carbono paralelos al eje del tubo. Las "nanofibras de carbono" (CNFs) son un término más general que se refiere a las estructuras de grafito con otras orientaciones del plano de grafeno, por lo que los CNTs se pueden considerar como un caso especial de las CNFs. Los nanotubos de carbono y nanofibras de carbono tienen muchas propiedades únicas, tales como alta resistencia a los ácidos y bases fuertes, alta conductividad eléctrica, alta área superficial y una alta resistencia mecánica Xie et al (2000). Estas propiedades hacen que losnanotubos de carbono posean un gran nuero de aplicaciones, tales como soportes de catalizadores, agentes de adsorción selectiva, almacenamiento de hidrógeno, refuerzo en materiales compuestos, dispositivos nanoelectrónicos y nano-mecánicos y dispositivos de emisión de campo, etc Lozano \& Barrera (2001).

Existen varios métodos para producir nanotubos de carbono, tales como descarga de arco Iijima (1991), ablación laser Kusaba et al (2006), deposición catalítica en fase de vapor (CVD) Li et al (1996), entre otros Terrones et al (1998). El método CVD normalmente es preferido debido a su simplicidad y a que permite la obtención de cantidades grandes de producto.

Los catalizadores para la producción de MWCNTs por el método CVD son usualmente basados en metales de transición ( $\mathrm{Fe}, \mathrm{Co}, \mathrm{Ni}$ ) soportados en óxidos inorgánicos. Hidrocarburos gaseosos como metano, etanol y ferroceno son usados como fuente de carbono Gallego et al (2011), Chaisitsak et al (2007). Pero por lo general la síntesis de los catalizadores es complicada y costosa ya que es necesario reactivos de alta calidad y condiciones especiales de síntesis. Sin embargo, el costo de producción de los nanotubos de carbono podría reducirse si se utiliza el método de CVD con un material natural como catalizador lo cual haría que el proceso fuera más amigable con el medio ambiente.

En este trabajo se presenta la síntesis de nanotubos de carbono de capa múltiple (MWCNTS) por el método de CVD usando $\mathrm{CH}_{4}$ como fuente de carbono y limonita como precursor catalítico.

\section{Metodología}

\subsection{Preparación del catalizador}

El mineral limonita se empleó como catalizador. La muestra de limonita en polvo fue calcinada en atmosfera de aire a $800^{\circ} \mathrm{C}$ durante 7 horas. Para estos experimentos se utilizó mineral con un tamaño de partícula inferior a los $60 \mu \mathrm{m}$

\subsection{Reacción de descomposición de metano}

El diagrama esquemático del sistema de reacción se muestra en la Figura 1. La descomposición del metano fue llevada a cabo en flujo continuo en un reactor de cuarzo de $25 \mathrm{~mm}$ de diámetro interno a una temperatura de $700^{\circ} \mathrm{C}$ a presión atmosférica. 


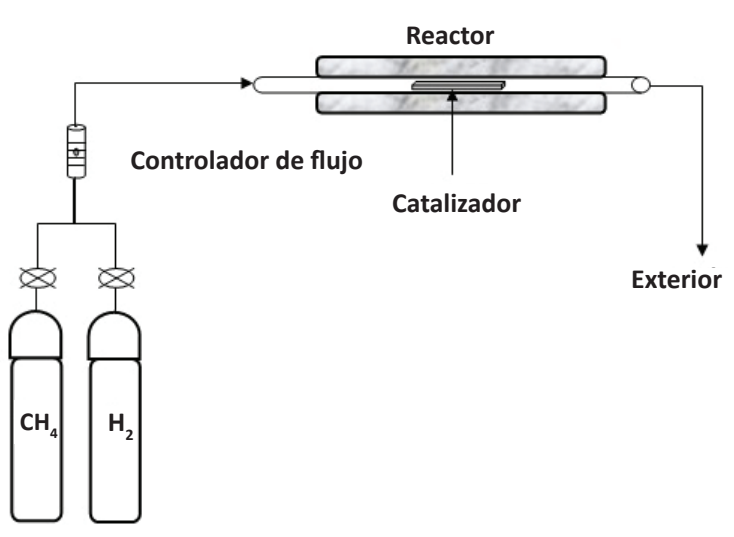

Figura 1. Diagrama esquemático del sistema de reacción

Aproximadamente $50 \mathrm{mg}$ del catalizador se introdujeron en el reactor y luego se sometió a tratamiento térmico bajo atmosfera de $\mathrm{H} 2(20 \mathrm{~mL} / \mathrm{min})$ a $700^{\circ} \mathrm{C}$ durante 60 minutos, con el objetivo de formar $\mathrm{Fe}^{\circ}$ metálico.

Luego de la reducción a $700^{\circ} \mathrm{C}, 20 \mathrm{~mL} / \mathrm{min}$ de CH4 (99.995\% de pureza) fueron introducidos al reactor a $700{ }^{\circ} \mathrm{C}$ y se mantuvo a esta temperatura por 4 horas. .

Los nanotubos de carbono obtenidos fueron purificados por tratamiento con acido para disolver el catalizador. Los MWCNTs fueron tratados con $\mathrm{H} 2 \mathrm{SO} 495.9 \%$, por una hora bajo continua agitación. El producto fue filtrado y lavado con agua des ionizada. El residuo fue luego secado a $100^{\circ} \mathrm{C}$ por $24 \mathrm{~h}$.

\subsection{Caracterización}

El catalizador fue caracterizado por difracción de rayos $X$ (DRX) usando un difractómetro de rayos $\mathrm{X}$ marca Palytical referencia $\mathrm{X}^{\prime} \mathrm{PERT}$ PRO MPD con ánodo cerámico de $\mathrm{Cu}$ con radiación $\mathrm{CuK} \alpha 1=1,5406 \AA$, operado con una potencia de $45 \mathrm{kV}$ y $40 \mathrm{~mA}$. Se hizo un barrido en 2 theta entre $4^{\circ}$ a $70^{\circ}$ con un paso de $0,013^{\circ}$ y un tiempo de 59 s/paso con rotación (spinning) de $90 \%$ s.

El análisis en el microscopio electrónico de transmisión (TEM) fue llevado a cabo en un equipo Philips CM120, con filamento de LaB6 y equipado con un analizador de rayos X (EDX). Las imágenes TEM fueron tomadas después de la purificación de los MWCNTs.
La microscopia electrónica de barrido (SEM) se llevó a cabo en un microscopio JEOL JSM 840.

Experimentos de TPR se realizaron en un equipo NETZSCH STA 409 CD TGA con $20 \mathrm{mg}$ de la muestra bajo atmosfera de hidrógeno a $50 \mathrm{~mL} / \mathrm{min}$ con velocidad de calentamiento de $10^{\circ} \mathrm{C} / \mathrm{min}$.

El análisis termogravimétrico (TGA) de los nanotubos de carbono se llevó a cabo en el mismo equipo NETZSCH STA 409 CD equipo TGA. Las muestras se colocaron en una porta muestras de alúmina en cantidades entre 5 a $8 \mathrm{mg}$ y se calentó a $10^{\circ} \mathrm{C} / \mathrm{min}$ desde temperatura ambiente hasta $900^{\circ} \mathrm{C}$ en atmósfera de aire.

\section{Resultados y discusión}

\subsection{Difracción de rayos $\mathrm{X}$ de la limonita}

El patrón de difracción de rayos X (DRX) para el mineral limonita calcinada antes y después de reducción es mostrado en la Figura 2.

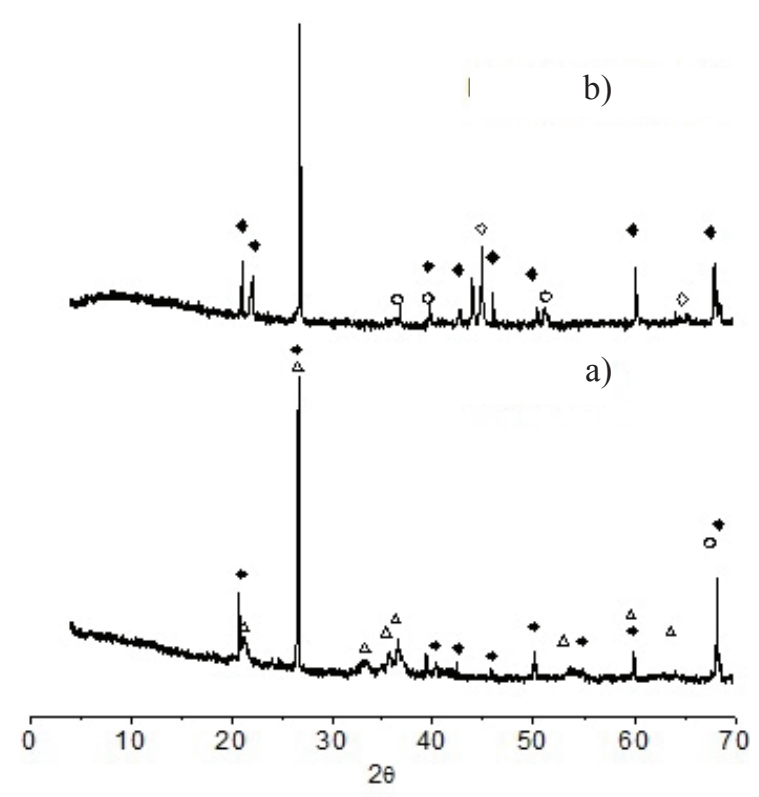

Figura 2. DRX a) limonita y b) limonita reducida. ( () $\mathrm{Fe}$, (৬) SiO2, (O) hematita Fe2O3 y ( $\Delta$ ) goethita.

El patrón de difracción reveló los picos característicos de la goethita y $\mathrm{SiO} 2$, lo que mostró que el mineral limonita se compone de una mezcla de estas dos fases principalmente. 
Después del tratamiento en atmosfera de hidrógeno a $700^{\circ} \mathrm{C}$, se observan las líneas de difracción características de la hematita y del $\mathrm{Fe}^{\circ}$.

Este resultado concuerda con el TPR (sección 3.2), mostrando que la limonita puede ser reducida parcialmente a una temperatura de $1200^{\circ} \mathrm{C}$ bajo atmosfera de $\mathrm{H}_{2}$ puro.

\subsection{Reducción a temperatura programada (TPR) de la limonita}

Experimentos de TPR se realizaron en un equipo NETZSCH STA 409 CD TGA con $20 \mathrm{mg}$ de la muestra bajo atmosfera de hidrógeno a $50 \mathrm{~mL} / \mathrm{min}$ con velocidad de calentamiento de $10^{\circ} \mathrm{C} / \mathrm{min}$.

El perfil de reducción a temperatura programada (TPR) del mineral limonita es presentado en la Figura 3, donde se observan dos eventos principales. El primer evento es pequeño y está centrado aproximadamente a $450^{\circ} \mathrm{C}$. Este evento corresponde a la modificación de la estructura de la goethita para dar una mezcla de hematita y hierro metálico, ver Ec. (1)

El segundo evento, es un pico ancho desde $520{ }^{\circ} \mathrm{C}$ hasta $900{ }^{\circ} \mathrm{C}$, el cual corresponde a varios eventos simultáneos: la continuación de la reducción parcial de la goethita para producir hematita, hierro metálico y agua; y a la reducción de la hematita para dar magnetita, metálico y agua Ec. (2).

Después de reducir a $1200^{\circ} \mathrm{C}$, se realizó difracción de rayos $\mathrm{X}$ (No presentados en este trabajo), los cuales mostraron la presencia de óxidos de hierro en la muestra reducida, lo que sugiere que solo una porción del hierro contenido en la goethita es reducido hasta $\mathrm{Fe}^{\circ}$, el resto permanece en la estructura de la hematita y magnetita. Sería necesario reducir a más de $1200^{\circ} \mathrm{C}$ para reducir totalmente la muestra mineral.

$$
\begin{gathered}
4 \alpha-\mathrm{FeOOH}_{(\mathrm{s})}+3 \mathrm{H}_{2(\mathrm{~g})} \rightarrow \alpha-\mathrm{Fe}_{2} \mathrm{O}_{3(\mathrm{~s})}+2 \mathrm{Fe}^{\circ}{ }_{(\mathrm{s})}+5 \mathrm{H}_{2} \mathrm{O}_{(\mathrm{g})} \\
4 \alpha-\mathrm{Fe}_{2} \mathrm{O}_{3(\mathrm{~s})}+8 \mathrm{H}_{2(\mathrm{~g})} \rightarrow \mathrm{Fe}_{3} \mathrm{O}_{4(\mathrm{~s})}+5 \mathrm{Fe}_{(\mathrm{s})}^{\circ}+8 \mathrm{H}_{2} \mathrm{O}_{(\mathrm{g})}
\end{gathered}
$$

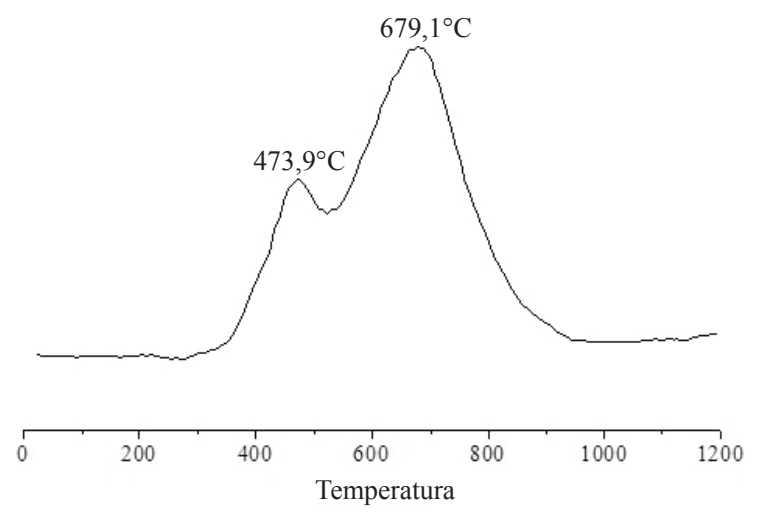

Figura 3. Perfil de reducción para el mineral limonita en $\mathrm{H}_{2}$ puro hasta $1200^{\circ} \mathrm{C}$

\subsection{Análisis elemental del mineral limonita}

La tabla1 muestra el análisis elemental del mineral limonita. En la limonita, el $\mathrm{Fe}$ es el mayor constituyente (alrededor del 25\% $\left.\mathrm{Fe}_{2} \mathrm{O}_{3}\right)$ seguido por $\mathrm{Si}\left(20 \% \mathrm{SiO}_{2}\right)$, Al (4\% $\mathrm{Al}_{2} \mathrm{O}_{3}$ ). Trazas de elementos como $\mathrm{Mg}, \mathrm{Zn}$ se encontraron también.

\subsection{Microscopía de barrido electrónica (SEM) de la limonita}

Las imágenes de microscopia electrónica de barrido (SEM) del mineral limonita son mostradas en la Figura 4. En esta Figura puede observarse que este mineral no tiene una superficie muy porosa. De igual forma se observan particulas esfericas de alrededor 500 micras.

\subsection{Microscopia electrónica de Barrido (SEM) y de transmisión (TEM) de los nanotubos de carbono}

Después de reacción los MWCNTs obtenidos fueron purificados por tratamiento con $\mathrm{H}_{2} \mathrm{SO}_{4}-95,9 \%$ para remover las partículas del catalizador. Después de esta purificación se realizaron los estudios de microscopia electrónica de barrido y transmisión. Las micrografías SEM se muestran en la Figura 5, 
Tabla 1. Análisis químico del mineral limonita

\begin{tabular}{cccccccc}
\hline Componente & $\mathrm{Fe}$ & $\mathrm{Si}$ & $\mathrm{Cu}$ & $\mathrm{Mg}$ & $\mathrm{Zn}$ & $\mathrm{Ni}$ & $\mathrm{Al}$ \\
\hline${\text { Wt. }{ }^{\mathrm{a}}{ }^{\mathrm{a}}}^{\mathrm{b}}$ & 21.13 & 17.5 & $<0.01$ & 0.4 & $<0.04$ & ---- & ---- \\
${\text { Wt. }{ }^{\mathrm{b}}}^{\mathrm{b}}$ & 28.08 & 24.85 & ------ & ---- & ----- & ---- & 4.11 \\
\hline
\end{tabular}

a: Medida por AA

${ }^{\mathrm{b}}$ : Medida por EDS
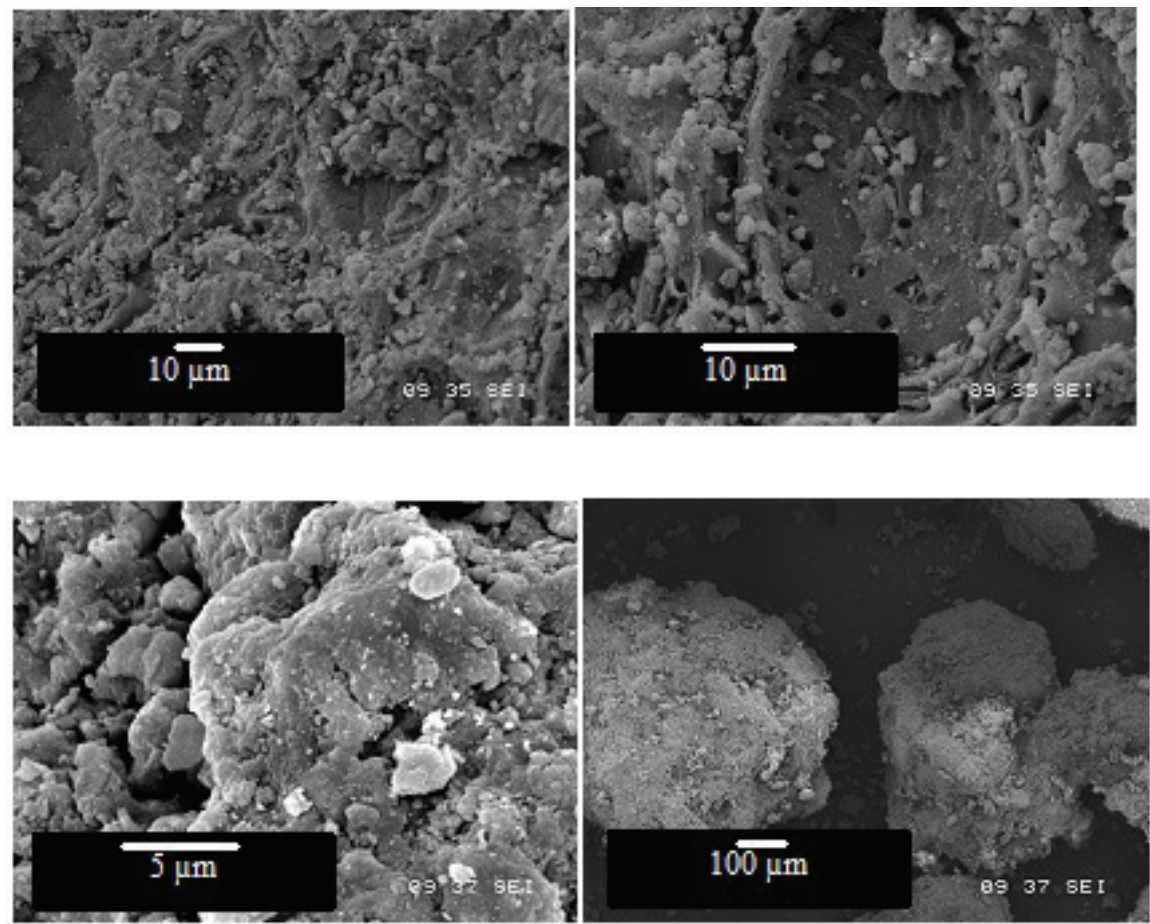

Figura 4. Microscopia obtenida en SEM del mineral limonita

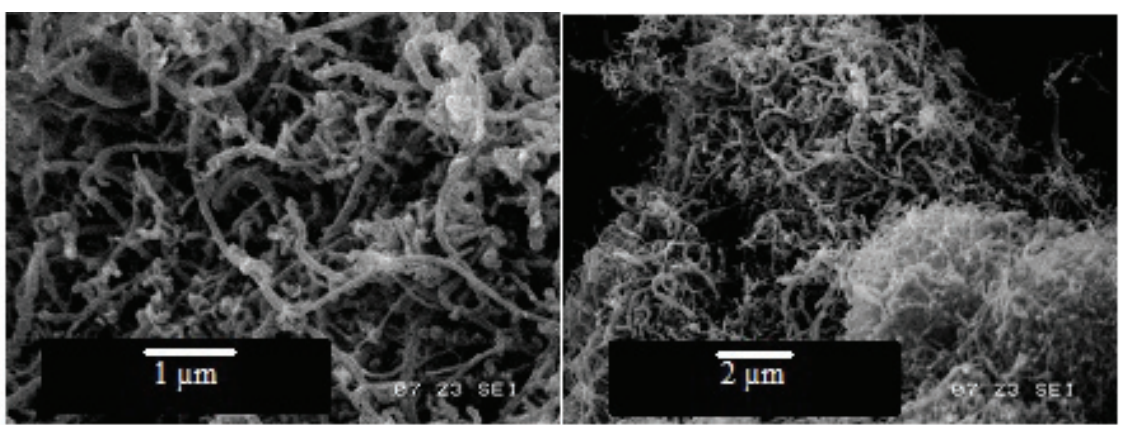

Figura 5. Micrografias SEM de los nanotubos de carbono multicapa después del tratamiento ácido

las cuales muestran la formación de filamentos carbonosos. Para verificar la formación de nanotubos de carbono se realizó Microscopía electrónica de transmisión (ver Figura 6). Las micrografías TEM proveen una clara evidencia de la formación de nanotubos multicapa durante la reacción de descomposición de metano, los cuales poseen varias micras de longitud.
Los diámetros interiores van desde $11 \mathrm{~nm}$ a 28 nm y un diámetro exterior de hasta alrededor de $47 \mathrm{~nm}$. En estas micrografías electrónicas se observaron partículas metálicas encapsuladas, tal como se ha reportado en la literatura para el mecanismo de crecimiento durante el método CVD, Sierra et al (2010). 

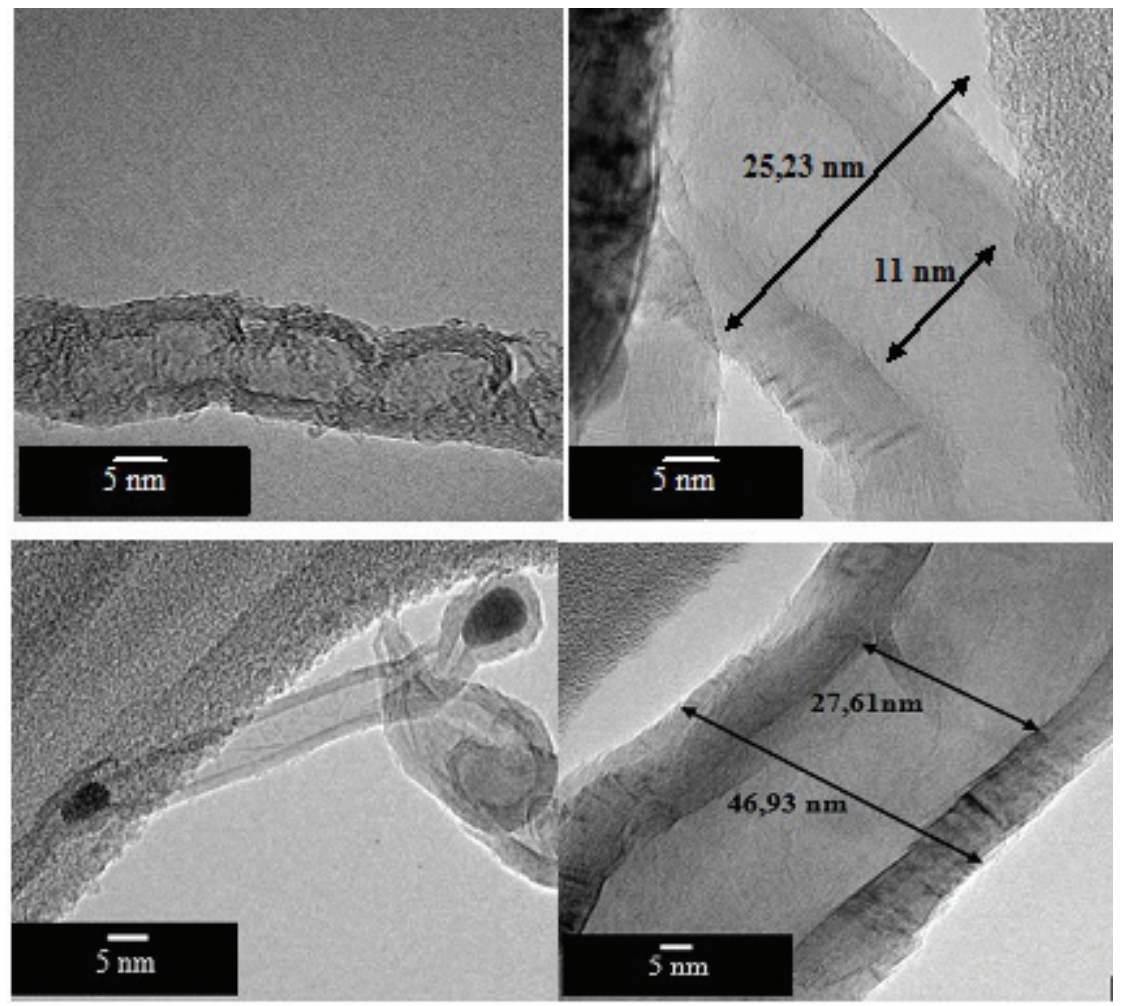

Figura 6. Micrografías SEM y TEM de los nanotubos de carbono multicapa después del tratamiento ácido

\subsubsection{Caracterización termogravimétrica de los MWCNTs}

Después de purificación con acido un análisis termogravimétrico fue llevado a cabo para estudiar el comportamiento en la oxidación de los MWCNTs. El análisis se realizó con una velocidad de calentamiento de $10^{\circ} \mathrm{C} / \mathrm{min}$ hasta $800^{\circ} \mathrm{C}$ en atmosfera de aire. El perfil de la oxidación se presenta en la Figura 7.

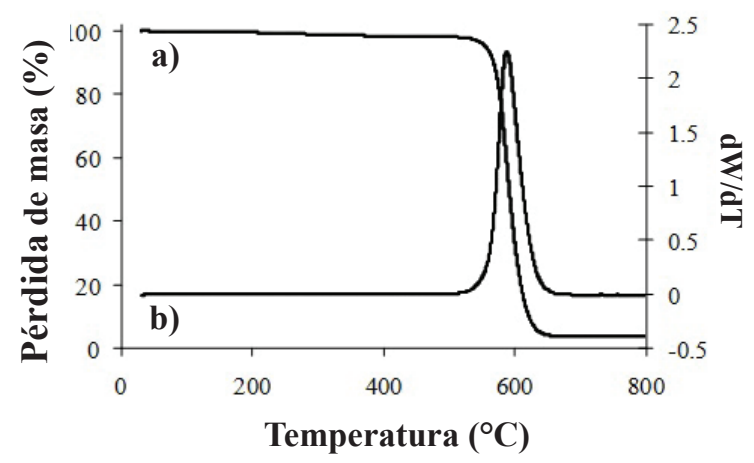

Figura 7. (a) TGA de los MWCNTs después de purificación (b) derivada de la pérdida de peso respecto a la temperatura
Una pérdida inicial del $0.5 \%$ ocurre desde temperatura ambiente hasta los $200{ }^{\circ} \mathrm{C}$, la cual es atribuida a la remoción de agua fisisorbida o $\mathrm{CO}_{2}$. Una segunda pérdida del $3.5 \%$ se observa hasta los $550{ }^{\circ} \mathrm{C}$, la cual puede ser atribuida a la oxidación de carbono amorfo conteniendo especies CHx o nanotubos con defectos en su estructura Djaidja et al (2006), Misra et al (2006). Esta pérdida es muy pequeña sugiriendo que la cantidad de carbono con estructuras desordenadas es muy baja.

La oxidación total de los nanotubos de carbono ocurre entre los 550 y $620^{\circ} \mathrm{C}$. Después de la oxidación aproximadamente un $5 \%$ de la masa original queda como residuo, proveniente tal vez de las partículas metálicas que quedaron encapsuladas en el carbón y que no pudieron ser disueltas con el ácido. La derivada de la pérdida de peso presenta un solo evento de oxidación con un máximo a los $590{ }^{\circ} \mathrm{C}$. Esta temperatura de oxidación de los MWCNTs $\left(590^{\circ} \mathrm{C}\right)$ es menor a la reportada por Ajayan de $700^{\circ} \mathrm{C}$ para MWCNTs preparados por descarga de arco Ajayan et al (1993), y mucho mayor 
que la reportada por Kukovitskii de $420^{\circ} \mathrm{C}$ para MWCNTs preparados por descomposición de propileno Kukovitskii et al (1997). La pureza de los MWCNTs fue estimada alrededor de 96\% ya que durante los procesos de purificación no hubo pérdida de peso, y en la oxidación con aire en el TGA perdió menos del $4 \%$ hasta los $500^{\circ} \mathrm{C}$.

\section{Conclusiones}

El mineral limonita después del proceso de reducción con $\mathrm{H} 2$ mostró actividad catalítica para la descomposición del metano hacia nanotubos de carbono. Los nanotubos obtenidos fueron multicapa, con diámetros internos que van desde $11 \mathrm{~nm}$ a $28 \mathrm{~nm}$ y diámetro exterior de hasta $47 \mathrm{~nm}$ como se observó en las micrografías TEM.

Análisis TGA mostró que la pureza de los MWCNTs era aproximadamente del $96 \%$, y su temperatura de oxidación esta alrededor de $590{ }^{\circ} \mathrm{C}$. Esta temperatura de oxidación sugiere que los MWCNTs poseen un alto grado de grafitación y orden estructural comparados con los MWCNTs preparados por métodos catalíticos reportados en la literatura.

\section{Agradecimientos}

Los autores agradecen a la Universidad Nacional de Colombia y a la Fundación para la Promoción de la Investigación y la Tecnología del Banco de la República por la financiación el proyecto "MODIFICACIÓN DEL COEFICIENTE DE FRICCIÓN ENTRE ACEROS INOXIDABLES USANDO NANOTUBOS DE CARBONO OBTENIDOS POR VIA CATALÍTICA" con código 2.668 .

\section{Referencias bibliográficas}

Ajayan, P., Ebbesen, T., Ichihashi, T., Iijama, S., Tanigaki, K., \& Hiura, H. (1993). Opening carbon nanotubes with oxygen and implications for filling. Nature 362 (6420), 522-525.

Chaisitsak, S., Nukeaw, J., \& Tuantranont, A. (2007). Parametric study of atmospheric- pressure single-walled carbon nanotubes growth by ferrocene-ethanol mist CVD. Diamond and Related Materials 16 (11), 1958-1966.

Djaidja, A., Libs, S., Kiennemann, A., \& Barama, A. (2006). Characterization and activity in dry reforming of methane onNiMg/ $\mathrm{Al}$ and $\mathrm{Ni} / \mathrm{MgO}$ catalysts. Catalysis Today 113 (3-4), 194-200.

Gallego, J., Sierra, G., Mondragon, F., Barrault, J., \& Batiot-Dupeyrat, C. (2011).Synthesis of MWCNTs and hydrogen from ethanol catalytic decomposition over a $\mathrm{Ni} / \mathrm{La}_{2} \mathrm{O}_{3}$ catalyst produced by the reduction of $\mathrm{LaNiO}_{3}$. Applied Catalysis A: General 397 (1-2), 73-81.

Kaneko, T., Sugita, S., Tamura, M., Shimasaki, K., Makino, E., \& Silalahi, L. (2002). Highly active limonite catalysts for direct coal liquefaction. Fuel 81 (11-12), 1541-1549.

Kukovitskii, E., \& Chernoszatonskii, L., L'vov, S., \& Mel'nik, N. (1997). Carbon nanotubes of polyethylene. Chemical Physics Letters 266 (34), 323-328.

Li, W., Xie, S., Qian, L., Chang, B., Zou, B., Zhou, W., Zhao, R., \&Wang, G. (1996). Largescale synthesis of aligned carbon nanotubes. Science 274 (5293), 1701-1703.

Lijima. S. (1991). Helical microtubules of graphitic carbon. Nature 354, 56-58

Lozano, K., \& Barrera, E. (2001). Nanofiberreinforced thermoplastic composites. I. Thermoanalytical and mechanical analyses. Journal of Applied Polymer Science 79 (1), 125-133.

Matsumura, A., Sato, S., Kondo, T., Saito, I. \& Souza, W. (2005). Hydrocracking Marlim vacuum residue with natural limonite. Part 2: Experimental cracking in a slurry-type continuous reactor. Fuel 84 (4), 417-421.

Misra, A., Tyagi, P., Singh, M., \& Misra, D. (2006). FTIR studies of nitrogen doped carbon nanotubes. Diamond and Related Materials 15 (2-3), 385-388. 
Sierra, G., Barrault, J., Batiot-Dupeyrat, C., \& Mondragón, F. (2010). Production of hydrogen and MWCNTs by methane decomposition over catalysts originated from $\mathrm{LaNiO}_{3}$ perovskite. Catalysis Today 149 (3-4), 365-371.

Souza, W., Guimarães, I., Oliveira, L., Guerreiro, M., Guarieiro, A., \& Carvalho, K. (2007). Natural and $\mathrm{H}_{2}$-reduced limonite for organic oxidation by a Fenton-like system: Mechanism study via ESI-MS and theoretical calculations. Journal of Molecular Catalysis A: Chemical 278 (1-2), 145-151.

Terrones, M., Grobert, N., Olivares, J., Zhang, J., Terrones, H., Kordatos, K., Hsu, W., Hare, J., Townsend, P., Prassides, K., Cheetham, A., Kroto, H., \& Walton, D. (1998). Preparation of aligned carbon nanotubes catalysed by laser- etched cobalt thin films. Chemical Physics Letters 285 (5-6), 299-305.

Kusaba, M., Tsunawaki, Y., (2006). Production of single-wall carbon nanotubes by a $\mathrm{XeCl}$ excimer laser ablation. Thin Solid Films 26 (506-507), 255-258.

Tsubouchi, N., Hashimoto, H., \& Ohtsuka, Y. (2008). Sulfur tolerance of an inexpensive limonite catalyst for high temperature decomposition of ammonia. Powder Technology 180 (1-2), 184-189.

Xie, S., Li, W., Pan, Z., Chang, B. \& Sun, L. (2000). Mechanical and physical properties on carbon nanotube. Journal of Physics. and Chemistry of solids 61 (7), 1153-1158. 